\title{
Outage Analysis of Cognitive Communication System Using Opportunistic Relay Selection and Secondary Users as Relays
}

Hojjat Javadzadeh

Kermanshah University of Technology

abdulhamid zahedi ( $\nabla$ zahedi@kut.ac.ir)

kermanshah university of technology https://orcid.org/0000-0003-4809-984X

\section{Research Article}

Keywords: Outage probability, Opportunistic relay selection, Secondary user, Primary user, Best relay.

Posted Date: May 7th, 2021

DOl: https://doi.org/10.21203/rs.3.rs-490647/v1

License: (c) (i) This work is licensed under a Creative Commons Attribution 4.0 International License.

Read Full License 


\title{
Outage Analysis of Cognitive Communication System Using Opportunistic Relay Selection and Secondary Users as Relays
}

\author{
Hojjat Javadzadeh, Abdulhamid Zahedi ${ }^{*}$ \\ Department of Electrical Engineering, Kermanshah University of Technology, Kermanshah, Iran
}

\begin{abstract}
Efficient bandwidth utilization is significant in new communication systems where secondary users can be used besides of primary users considering interference issues and idle state of primary users. Using secondary users as relays to transmit their own signals in addition to the primary signals can be applied for more reliability of the system where opportunistic relay selection can significantly enhance the performance of the system. The best-condition secondary user is selected as the optimum relay for retransmission of primary/secondary signal. Outage probability is analyzed in this paper based on decode and forward techniques in secondary users while the closed-form statement for outage probability is provided and verified by numerical evaluations.
\end{abstract}

Keywords- Outage probability, Opportunistic relay selection, Secondary user, Primary user, Best relay.

\section{Introduction}

Nowadays, Higher demands of different wireless technologies with high bandwidth requirement causes the focus on the efficient exploitation of spectrum in communication systems. Cognitive communication approach is a promising solution for better using and prevent underutilization of spectrum. Unused spectrum of primary users (PUs) can be exploited by secondary users (SUs) to transmit their data in the spectrum when PU is idle. On the other hand, to improve the capacity and data rate of the communication system, relay-based strategies can be applied as an interface for more reliable data delivery and throughput. In these cooperative systems, multiple relays can play the interface role in delivering the data from sender node to the destination one. However, using multiple relays can affect the spectral efficiency where using this strategy needs more time slots to prevent the interference between relay signals and transmitter/receiver signals. For $M$ assumed relays, $M+1$ time slots must be used where the direct link is assumed in the system. Thus, selecting the best relay can increase the spectral efficiency in which one best relay based on signal-to-noise ratio (SNR) is selected for the relaying the signal from source node. Therefor two time-slot, one for direct link and another for best-relay node is required for the signal transmission in this system which is called opportunistic relay approach.

Combining the cognitive strategy and cooperative approach, multiple secondary users can be assumed as multiple relays in which in the first time slot, they receive the signal of primary user and in the second time slot, they can relay the PU signal in addition to the transmission of their own signal. Also for more spectral efficiency, one best relay is selected to repeat the PU signal and its own signal. The strategy of signal relaying is considered Decode and Forward (DF). The

\footnotetext{
${ }^{*}$ Corresponding Author, Email: zahedi@kut.ac.ir
} 
main purpose of this structure is selecting the best relay based on SNR criterion to improve the reliability of the communication system and the spectral efficiency. Also, the outage probability is the main criterion for evaluating the efficiency of the proposed system where in different conditions, the outage must be lower than predefined threshold for the reliability and satisfaction of communication systems.

\section{Literature Review}

The outage probability analysis of cognitive transmissions by considering the two phases as the first phase for spectrum hole detection and the second phase for data transmission is discussed in [1]. Closed-form expression for outage probability is achieved with consideration of the probability of no spectrum hole detection and the cognitive transmissions over Rayleigh fading channels. Optimal spectrum sensing is also applied to minimize the outage probability [1]. In [2], the optimum secondary user power allocation to improve the ergodic multicast rate of point-tomultipoint communication in a cognitive radio network is presented with the assumption of outage constraints for the primary users. Weibull distribution is considered for outage analysis when the user signal and the interferer signals experience independent non-identically distributed (i.n.i.d.) $\mathrm{k}-\mu$ shadowed fading [2]. Improper Gaussian signaling effect is applied in [3] which is compared to conventional Gaussian signaling to enhance the achievable rate of systems that suffer from interference. Underlay cognitive radio is assumed in which discussing the achievable outage probability of both the primary user and secondary user is presented. Physical layer security based on existence of multiple eavesdroppers in 5G network is considered in [4] where outage and capacity analysis is performed in this paper. $5 \mathrm{G}$ network is assumed in cognitive radio status in IoT-based system with consideration of $\kappa-\mu$ shadowed fading. Non-Orthogonal Multiple Access (NOMA) system in cognitive radio is assumed in [5] to enhance the capacity and outage probability. Two cases of single-antenna and multiple-antenna are analyzed for throughput maximization. Security analysis considering outage probability in existence of eavesdroppers is discussed in [6]. Multiple eavesdroppers are assumed to interfere with secondary and primary users in assumption of Nakagami-m fading channel where channel cascading degree effect is considered in the paper. Performance analysis based on throughput analysis is realized in [7] for two cognitive systems as interweaved and underlay multiple-inputsingle-output (MISO) systems. The achievable ergodic capacity of cognitive system is compared in two strategies. The throughput analysis of two cognitive systems is presented in [8] where sensing criterion and probability of true sensing effect on throughput are analyzed. In [9], outage probability is presented in cognitive relay networks, in which the best relay is selected based on full and partial channel state information. Closed-form statement for outage analysis is obtained in this paper. Outage probability is presented in cognitive radio communication based on energy harvesting technique [10]. Reinforcement learning and multi-hop Q-routing is applied in this cognitive system with cooperative spectrum sensing. Optimization of power allocation and time of energy harvesting in secondary user for outage minimization is done in [10]. Device-to-device (D2D) transmission together with traditional communication is presented in [11] to form a new system model in NOMA. The perfect Successive Interference Cancellation (SIC) is assisted to cancel the interference. In [12], the imperfect information of channel of SU to PU is assumed where outage probability is considered as the main quality parameter for cognitive radio system evaluation. Presence of PU interference in cognitive system and interference analysis is done in [13] to discuss the outage probability in decode and forward (DF) strategy. Secondary multirelay network using decode-and-forward scheme and opportunistic best-relay selection is 
proposed in [14] subjected to the constraints of the spectrum-sharing and interference. The closed-form expression of the outage probability of secondary user is presented in this paper. Relay-based communication with opportunistic relay selection strategy in existence of direct link is also discussed in [15] for the analysis of outage probability. Cooperative scheme with selective relay strategy in dissimilar Rayleigh fading channels is assumed in [16, 17]. Moreover, relay selection strategy with optimal approach to maximize capacity in existence of interference and channel state information is discussed in [18]. Secondary rate maximization with considering the constraint of primary rate is presented in [19] subjected to the interference constraint and power constraint as the main conditions of optimization. Outage probability and effective capacity in overlay and underlay strategy are discussed for maximization of effective capacity and minimization of outage in [20]. Spectrum sharing in cognitive system considering multiple antennas in PU/SU transmitters is assumed in [21]. DF strategy is considered as the effective relaying strategy in this paper.

Considering above, the opportunistic relay selection in cognitive cooperative system based on outage analysis is the main purpose of this paper. Selecting the best relay based on SNR improves the performance of the communication system in term of spectral efficiency and outage probability. Accurate analysis of outage probability in numerical evaluation and simulation result is presented to propose a new efficient scheme in the cognitive system where both results verify each other.

In the sequel of the paper, in section 3, the model of communication system is presented where in section 4, quality of service (QoS) analysis is stated. Numerical results are provided in section 5 where some conclusions are mentioned in the last section, section 6.

\section{System model}

In Figure 1, the system model is shown where secondary users paly the relay role in the system and transmit their own signals in addition to the primary user signal. The source and destination nodes of PU and SU are shown in this figure where $h$ is used for the channel of PU to SUs and $g$ is used for relay-destination channel.

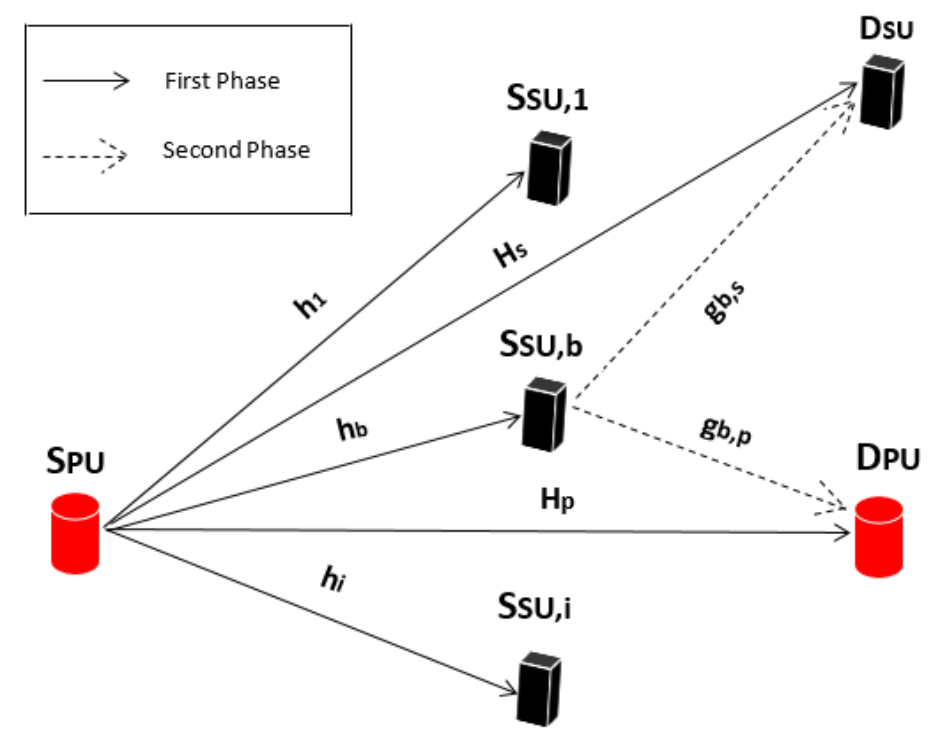

Figure 1. Proposed cognitive-based cooperative communication system 
The strategy of relaying is based on decode and forward where from the multiple SUs in the model, best-condition SU node is selected as the optimum relay to deliver the signal of PU to its destination. The index of su is used for SU nodes and pu is used for primary elements. The index $b$ is denoted for the best secondary node selected for signal relaying. $S_{s u}$ is the secondary sender node where $S_{p u}$ is the sender of primary user. The similar notation is used for destinations of SU and PU $\left(D_{s u}, D_{p u}\right) . h_{i}$ is the channel between $S_{p u}$ and $S_{S u, i}$ where $H_{p}$ is the direct link between $S_{P u}$ and $D_{p u}$. $H_{S}$ is also the direct link between $S_{P u}$ and $D_{S u}$ using which, the interference cancellation (PU signal) can be done in the secondary destination. $g_{i, P}$ is the channel gain between $S_{S u, i}$ and $D_{P u}$ where $g_{i, s}$ is the channel gain between $S_{S u, i}$ and $D_{s u}$.

The strategy of signal transmission from sender to the destination is that in the first phase, $S_{P u}$ can send its signal to the $D_{p u}$ and $D_{S u}$ (direct links) and to the multiple SU relays. If the channel in direct link is broken, the best relay of SUs can cooperate to bring the PU sender signal to its destination. The SU sender transmits both PU and SU signal. The channels in this model are independent but not identical (dissimilar). From $M$ relay nodes as SUs, several SUs can optimally receive and decode the PU signal which make the decode set as C. from this set, one relay with maximum SNR is selected for relaying the PU signal in the second phase.

In the first phase, the received signal of relays from PU is as:

$$
y_{S_{s u, i}}=\sqrt{P_{p}} x_{p} h_{i}+n_{i}
$$

For the PU destination in direct link, it can be written as:

$$
y_{D_{p u, 1}}=\sqrt{P_{\mathrm{p}}} x_{p} H_{P}+n_{p}
$$

and in the SU destination, we have:

$$
y_{D_{s u}}=\sqrt{P_{\mathrm{p}}} x_{p} H_{S}+n_{s}
$$

The SNR due to (1) is calculated as:

$$
\gamma_{h i}=\frac{P_{p}\left|h_{i}\right|^{2}}{N_{0}}
$$

Where $N_{0}$ is the Gaussian noise in relays. The SNR of direct link from PU to its destination is:

$$
\gamma_{1}=\frac{P_{p}\left|H_{P}\right|^{2}}{N_{0}}
$$

And also for another direct link, we have:

$$
\gamma_{2}=\frac{P_{p}\left|H_{s}\right|^{2}}{N_{0}}
$$

It is worth mentioning that general assumption of Gaussian noise, $N_{0}$ is assumed in each destination. In the second phase, the best secondary node is selected in which a part of power is allocated for PU signal transmission and another part is devoted for the secondary signal. The signal of relay in PU destination is presented as: 


$$
\mathrm{y}_{\mathrm{D}_{\mathrm{pu}, 2}}=\sqrt{\alpha \mathrm{P}_{\mathrm{s}}} x_{p} \mathrm{~g}_{\mathrm{b}, \mathrm{p}}+\sqrt{(1-\alpha) \mathrm{P}_{\mathrm{s}}} x_{s} \mathrm{~g}_{\mathrm{b}, \mathrm{p}}+n
$$

Also in SU destination, we have:

$$
y_{D_{s u}}=\sqrt{\alpha \mathrm{P}_{\mathrm{s}}} x_{p} \mathrm{~g}_{\mathrm{b}, \mathrm{s}}+\sqrt{(1-\alpha) \mathrm{P}_{\mathrm{s}}} x_{s} \mathrm{~g}_{\mathrm{b}, \mathrm{s}}+\mathrm{n}
$$

For the SNR calculation in $D_{p u}$, due to (7), we have:

$$
\gamma_{D_{p u, 2}}=\frac{\alpha \mathrm{P}_{\mathrm{s}}\left|\mathrm{g}_{\mathbf{b}, \mathbf{p}}\right|^{2}}{(1-\alpha) \mathrm{P}_{\mathrm{s}}\left|\mathrm{g}_{\mathbf{b}, \mathbf{p}}\right|^{2}+\mathrm{N}_{0}}
$$

The first term in denominator in (9) is denoted for the maximum tolerable interference from SU on PU signal in destination node which can be stated as:

Thus, (9) can be rewritten as:

$$
(1-\alpha) \mathrm{P}_{\mathrm{s}}\left|\mathrm{g}_{\mathrm{b}, \mathrm{p}}\right|^{2}=\mathrm{I}_{\mathrm{NT}}
$$

$$
\gamma_{D_{p u, 2}}=\frac{\alpha \mathrm{P}_{\mathrm{s}}\left|\mathrm{g}_{\mathbf{b}, \mathbf{p}}\right|^{2}}{\mathrm{I}_{\mathrm{NT}}+\mathrm{N}_{0}}
$$

The interference threshold must be chosen as the outage happening is prevented and the acceptable data rate is achieved as:

$$
\frac{1}{2} \log _{2}\left(1+\frac{\alpha \mathrm{P}_{\mathrm{s}}\left|\mathrm{g}_{\mathrm{b}, \mathrm{p}}\right|^{2}}{\mathrm{I}_{\mathrm{NT}}+\mathrm{N}_{0}}\right) \geq \mathrm{R}
$$

Thus, the interference can be presented as below:

$$
\mathrm{I}_{\mathrm{NT}}=\frac{\alpha \mathrm{P}_{\mathrm{s}}\left|\mathrm{g}_{\mathbf{b}, \mathbf{p}}\right|^{2}}{2^{2 \mathrm{R}}-1}-\mathrm{N}_{0}
$$

Moreover, the SNR in secondary destination due to (8) can be written as:

$$
\gamma_{\mathrm{D}_{\mathrm{su}, 2}}=\frac{(1-\alpha) \mathrm{P}_{\mathrm{s}}\left|\mathrm{g}_{\mathrm{b}, \mathrm{s}}\right|^{2}}{\alpha \mathrm{P}_{\mathrm{s}}\left|\mathrm{g}_{\mathrm{b}, \mathrm{s}}\right|^{2}+\mathrm{N}_{0}}
$$

Since the PU sends its signal for destination of secondary in the first phase, the interference of PU on SU can be suppressed and the SNR can be stated as:

$$
\gamma_{\mathrm{D}_{\mathrm{su}, 2}}=\frac{(1-\alpha) \mathrm{P}_{\mathrm{s}}\left|\mathrm{g}_{\mathrm{b}, \mathrm{s}}\right|^{2}}{\mathrm{~N}_{0}}
$$

\section{QoS analysis}

The probability of not qualifying of SU relay to be as a relay node can be described as:

$$
A_{i}=\operatorname{Pr}\left(\gamma_{h i} \leq 2^{2 R}-1\right)=1-\exp \left(-\frac{2^{2 R}-1}{\bar{\gamma}_{h i}}\right)
$$


$R$ is the threshold lower which the connection is blocked. The probability function of the channel from PU sender to its destination through the SU relay nodes can be described as below [15]:

$$
f_{y_{i}}(x)=f_{y_{i}} \mid\left(R_{i} \text { is down }\right) \operatorname{pr}\left(R_{i} \text { is down }\right)+f_{y_{i}} \mid\left(R_{i} \text { is active }\right) \operatorname{pr}\left(R_{i} \text { is active }\right)
$$

Thus, we can say:

$$
\begin{gathered}
\operatorname{pr}\left(R_{i} \text { is active }\right)=\left(1-A_{i}\right) \\
\operatorname{pr}\left(R_{i} \text { is down }\right)=A_{i}
\end{gathered}
$$

Thus, the probability distribution function (pdf) of dropped link is presented as:

$$
f_{y_{i} \mid R_{i}} \text { is down }(x)=\delta(x)
$$

and the pdf of active link from PU to SU nodes and then to the destination can be presented as:

$$
f_{y_{i} \mid R_{i} \text { is active }}(x)=\frac{1}{\bar{\gamma}_{g_{i}}} \exp \left(-x / \bar{\gamma}_{g_{i}}\right)
$$

Where $\bar{\gamma}$ presents the average SNR. Thus, combining these two links, we have:

$$
f_{y_{i}}(x)=A_{i} \delta(x)+\left(1-A_{i}\right) \frac{1}{\bar{\gamma}_{g_{i}}} \exp \left(-x / \bar{\gamma}_{g_{i}}\right) \quad i=1,2, \ldots, M
$$

\subsection{PU Outage Analysis}

Using maximum ratio combining (MRC) in PU destination, we have combined SNR of first and second phases as:

$$
I D F=\frac{1}{2} \log \left(1+\gamma_{1}+\max \left(\gamma_{D_{p u, 2}}\right)\right)
$$

Where IDF is used for the capacity of the PU destination. For analysis of PU outage probability, we have:

$$
p_{\text {out }, p u}=p(I D F<R)=p\left(\gamma_{1}+\max \left(\gamma_{D_{p u, 2}}\right)<2^{2 R}-1\right)
$$

Which leads to convolution of each PDF of direct and indirect link as [15, 21]:

$$
p_{\text {out }, p u}=p\left(\gamma_{1}<2^{2 R}-1\right) * p\left(\max \left(\gamma_{D_{p u, 2}}\right)<2^{2 R}-1\right)
$$

Resulting in:

$$
\begin{gathered}
p_{\text {out }, p u}=\left(\prod_{i=1}^{M} A_{i}\right)\left(1-\exp \left(\frac{-x}{\bar{\gamma}_{1}}\right)\right)+ \\
\sum_{k=1}^{M}(-1)^{k+1} \sum_{\lambda_{1}}^{M-k+1} \sum_{\lambda_{2}=\lambda_{1}+1}^{M-k+2} \ldots \sum_{\lambda_{k}=\lambda_{k-1}+1}^{M} \prod_{i=1}^{k}\left(1-A_{\lambda_{i}}\right) \times
\end{gathered}
$$




$$
\left[1+\frac{1}{\bar{\gamma}_{1}-1 / \sum_{i=1}^{k} \frac{1}{\bar{\gamma}_{D_{p u, 2, \lambda_{i}}}}}\left(\frac{\prod_{i=1}^{k} \exp \left(-x /_{D_{p u, 2, \lambda_{i}}}\right)}{\sum_{i=1}^{k} \frac{1}{\bar{\gamma}_{D_{p u, 2, \lambda_{i}}}}}-\bar{\gamma}_{1} \exp \left(\frac{-x}{\bar{\gamma}_{1}}\right)\right)\right]
$$

\subsection{SU Outage Analysis:}

To prevent outage happening for SU, SU destination must receive the SU sender signal in addition to the PU signal in the first phase. Thus, the outage probability can be stated as:

$$
p_{\text {out }, \text { su }}=1-\operatorname{Pr}\left(\max \left(\gamma_{\mathrm{D}_{\mathrm{su}, 2}}\right)>2^{2 R}-1\right) \times \operatorname{Pr}\left(\frac{1}{2} R_{2}>R\right)
$$

Where $R_{2}$ is the rate between primary transmitter and secondary receiver in the first phase described as $R_{2}=\frac{1}{2} \log _{2}\left(1+\gamma_{2}\right)$. The reason of applying the factor of $\frac{1}{2}$ is that the PU signal is transmitted in the first time slot to the destination of SU (the whole transmission needs two time slots). Then each term of (25) can be presented to calculate the total outage probability. For the first term, we have:

$$
p\left(\max \left(\gamma_{\mathrm{D}_{\mathrm{su}, 2}}\right)>2^{2 R}-1\right)=1-p\left(\max \left(\gamma_{\mathrm{D}_{\mathrm{su}, 2}}\right)<2^{2 R}-1\right)
$$

Where we have:

$$
p\left(\max \left(\gamma_{\mathrm{D}_{\mathrm{su}, 2}}\right)<2^{2 R}-1\right)=\int_{0}^{x} f_{x}(x) d x
$$

In other hand, we have the $\operatorname{pdf}\left(f_{x}(x)\right)$ as $[15,21]$ :

$$
\begin{aligned}
f_{x}(x)=\left(\prod_{i=1}^{M} A_{i}\right) & \delta_{(x)} \\
& +\sum_{k=1}^{M}(-1)^{k+1} \sum_{\lambda_{1}}^{M-k+1} \sum_{\lambda_{2}=\lambda_{1}+1}^{M-k+2} \ldots \sum_{\lambda_{k}=\lambda_{k-1}+1}^{M} \prod_{i=1}^{k}\left(1-A_{\lambda_{i}}\right) \\
& \times \exp \left(-x / \bar{\gamma}_{\mathrm{D}_{\mathrm{su}, 2}, \lambda_{i}}\right) \sum_{i=1}^{k} \frac{1}{\bar{\gamma}_{\mathrm{D}_{\mathrm{su}, 2}, \lambda_{i}}}
\end{aligned}
$$

Therefore, due to (27) and (28), the result can be stated as:

$$
\begin{gathered}
\operatorname{Pr}\left(\max \left(\gamma_{\mathrm{D}_{\mathrm{su}, 2}}\right)<2^{2 R}-1\right)= \\
\left(\prod_{i=1}^{M} A_{i}\right)+\sum_{k=1}^{M}(-1)^{k+1} \sum_{\lambda_{1}}^{M-k+1} \sum_{\lambda_{2}=\lambda_{1}+1}^{M-k+2} \ldots \sum_{\lambda_{k}=\lambda_{k-1}+1}^{M} \prod_{i=1}^{k}\left(1-A_{\lambda_{i}}\right)
\end{gathered}
$$




$$
\times\left(1-\exp \left(-x / \bar{\gamma}_{\mathrm{D}_{\mathrm{su}, 2}, \lambda_{i}}\right)\right)
$$

And for the second term, we can write as:

$$
\begin{gathered}
p\left(\frac{1}{2} R_{2}>R\right)=\operatorname{Pr}\left(\frac{1}{2} \log _{2}\left(1+\gamma_{2}\right)>R\right)=\operatorname{Pr}\left(\gamma_{2}>\frac{2^{2 R}-1}{\bar{\gamma}_{2}}\right) \\
=\exp \left(-\frac{2^{2 R}-1}{\bar{\gamma}_{2}}\right)
\end{gathered}
$$

Therefore, the outage probability of SU can be written as:

$$
\begin{aligned}
p_{\text {out }, \text { su }}= & 1-\left(\prod_{i=1}^{M} A_{i}\right)+\sum_{k=1}^{M}(-1)^{k+1} \sum_{\lambda_{1}}^{M-k+1} \sum_{\lambda_{2}=\lambda_{1}+1}^{M-k+2} \ldots \sum_{\lambda_{k}=\lambda_{k-1}+1}^{M} \prod_{i=1}^{k}\left(1-A_{\lambda_{i}}\right) \\
& \left.\times\left(1-\exp \left(-x / \bar{\gamma}_{\mathrm{D}_{\mathrm{su}, 2}, \lambda_{i}}\right)\right)\right) \times \exp \left(-\frac{2^{2 R}-1}{\bar{\gamma}_{2}}\right)
\end{aligned}
$$

\section{Numerical Results}

In this section, the numerical evaluation of above discussion is provided to verify the theoretical analysis of previous section. In the first analysis, the outage probability in different number of secondary users is presented versus change of SNR. As depicted in Figure 2, the outage probability is reduced with increasing the number of relays which is predictable in the proposed cognitive system. In addition, without any secondary user, the outage probability depends only on primary channel which is constant versus SNR.

In the sequel, the analysis of outage probability versus different outage thresholds for outage happening is described where the results are shown in Figure 3. As depicted in this figure, increasing the threshold causes more outage in the proposed system while reducing the threshold leads to outage probability reduction. This result shows the effect of selection of the threshold of channel condition on the outage probability.

In the third test case, the secondary user outage probability is discussed and its result is depicted in Figure 4. As described in this figure, using multiple Relays and selection of lower threshold causes less outage probability in the proposed system. It is obvious that increasing number of secondary users as relays improves the outage probability. 


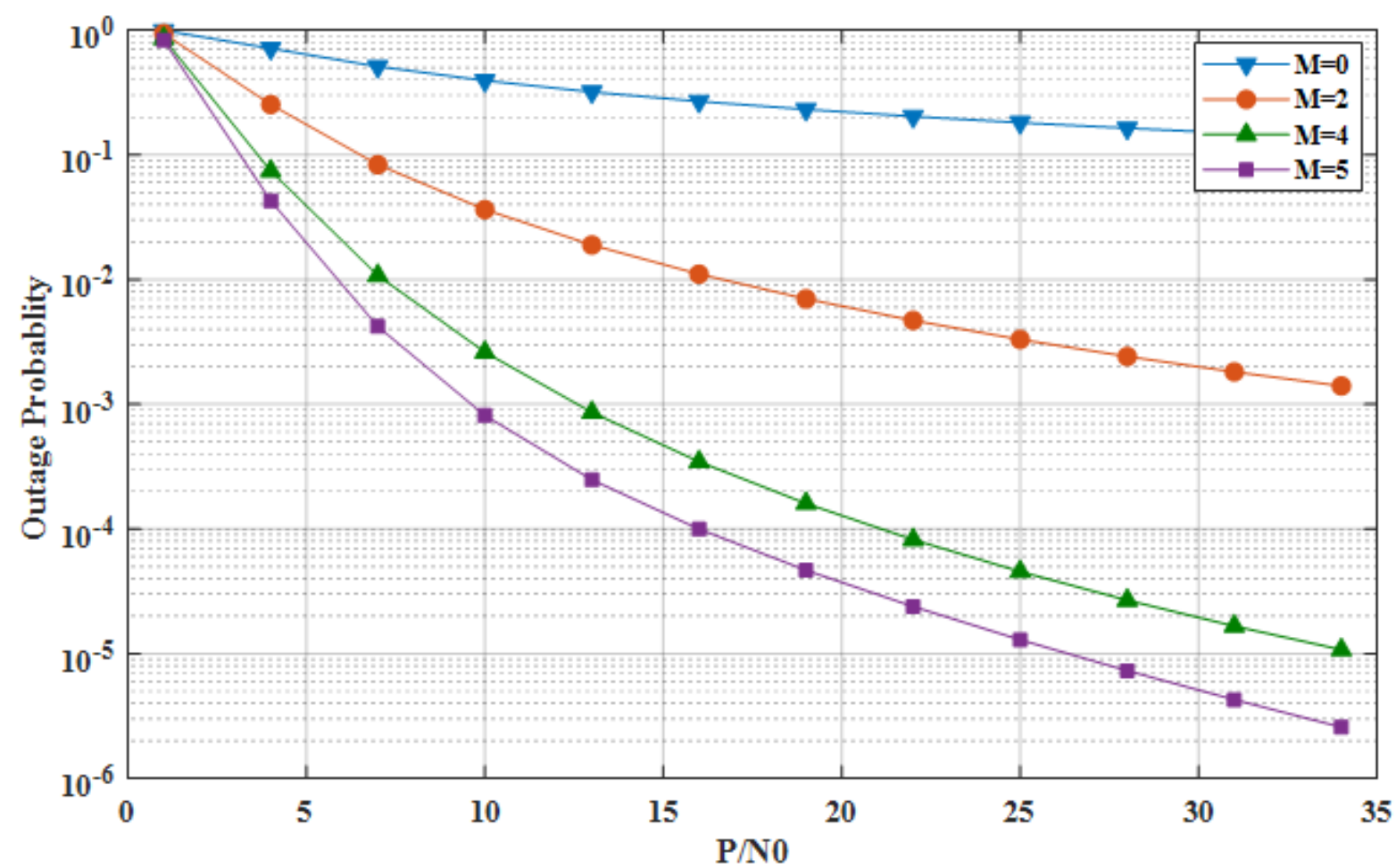

Figure 2. Outage probability of PU versus the SNR in different number of relays

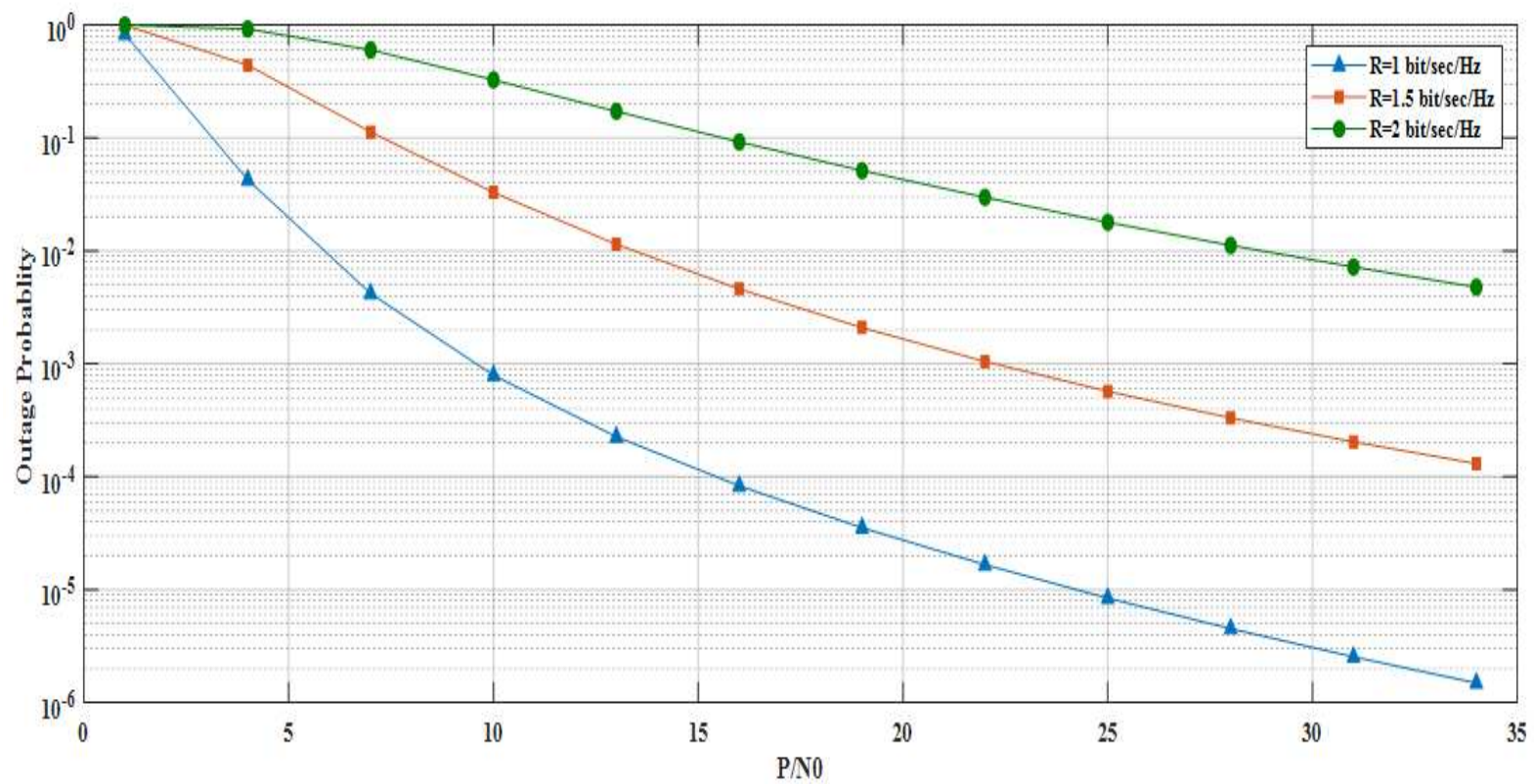

Figure 3. Outage probability of PU versus the SNR in different outage threshold 


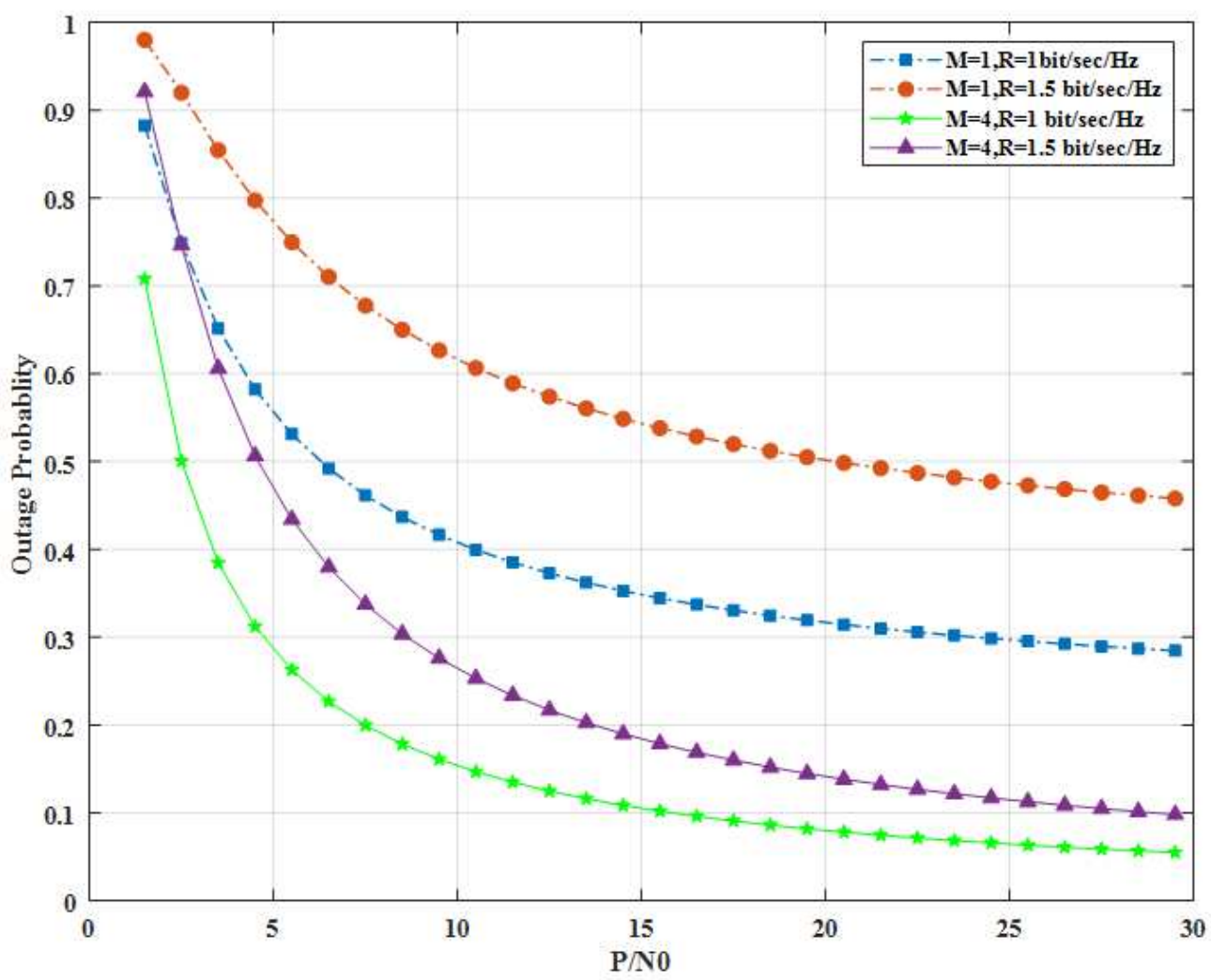

Figure 4. Outage probability of SU versus the SNR in different number of relays and outage thresholds

\section{Conclusion}

In this paper, the outage analysis of cognitive cooperative communication system based on multiple secondary users as relays and using opportunistic relay selection for retransmission is provided. Selection of the best relay from $M$ assumed relays causes more spectral efficiency which can improve the reliability and reduce outage probability. Theoretical analysis is discussed and evaluated using simulation results where both outcomes verify each other. Increasing multiple relays and reducing threshold of selection of best relay cause outage probability to be reduced more and thus, the performance of the proposed cognitive system is significantly improved.

\section{Conflict of interest statement}

On behalf of all authors, the corresponding author states that there is no conflict of interest.

\section{References}

[1] Y. Zou, Y. Yao and B. Zheng, "Outage Probability Analysis of Cognitive Transmissions: Impact of Spectrum Sensing Overhead," in IEEE Transactions on Wireless Communications, vol. 9, no. 8, pp. 26762688, August 2010, doi: 10.1109/TWC.2010.061710.100108.

[2] A. Subhash, M. Srinivasan, S. Kalyani and L. Hanzo, "Transmit Power Policy and Ergodic Multicast Rate Analysis of Cognitive Radio Networks in Generalized Fading," in IEEE Transactions on Communications, vol. 68, no. 6, pp. 3311-3325, June 2020, doi: 10.1109/TCOMM.2020.2980843. 
[3] O. Amin, W. Abediseid and M. Alouini, "Underlay Cognitive Radio Systems with Improper Gaussian Signaling: Outage Performance Analysis," in IEEE Transactions on Wireless Communications, vol. 15, no. 7, pp. 4875-4887, July 2016, doi: 10.1109/TWC.2016.2547918.

[4] Xu, Lingwei, Sun, Jiangfeng, Bie, Zhisong, Bie, Hongxia, He, Pengfei, Jin, Machao, Security and Communication Networks, 2020, "Secrecy Analysis of Cognitive Radio Networks over Generalized Fading Channels", SP. 8842012, vol. 2020, https://doi.org/10.1155/2020/8842012.

[5] TRAN, Thanh-Nam, Dinh-Thuan DO a Miroslav VOZŇÁK. On outage probability and throughput performance of cognitive radio inspired noma relay system. Advances in Electrical and Electronic Engineering. 2018, 16(4), 501-512.

[6] Ata, SÖ, Erdogan, E. Secrecy outage probability of inter-vehicular cognitive radio networks. Int J Commun Syst. 2020; 33: e4244. https://doi.org/10.1002/dac.4244

[7] M. C. Filippou, D. Gesbert and G. A. Ropokis, "A Comparative Performance Analysis of Interweave and Underlay Multi-Antenna Cognitive Radio Networks," in IEEE Transactions on Wireless Communications, vol. 14, no. 5, pp. 2911-2925, May 2015, doi: 10.1109/TWC.2015.2396519.

[8] Qian, Lijun, Omotere, Oluwaseyi, Jantti, Riku, "Performance Analysis on the Coexistence of Multiple Cognitive Radio Networks", vol. 1, DO - 10.4108/cogcom.1.2. e1, EAI Endorsed Transactions on Cognitive Communications.

[9] JOUR, Dong, Li, SP - 2730-2735, "Outage Probability of Cognitive Radio Networks with Relay Selection”, VL - 5, DO - 10.1049/iet-com.2011.0216, JO - IET Communications, 2011.

[10] A. Paul and S. P. Maity, "Outage Analysis in Cognitive Radio Networks with Energy Harvesting and Q-Routing," in IEEE Transactions on Vehicular Technology, vol. 69, no. 6, pp. 6755-6765, June 2020, doi: 10.1109/TVT.2020.2987751.

[11] Do, D.-T.; Nguyen, M.-S.V.; Lee, B.M. Outage Performance Improvement by Selected User in D2D Transmission and Implementation of Cognitive Radio-Assisted NOMA. Sensors 2019, 19, 4840.

[12] Toan, H., Bao, V. \& Le, K. Performance analysis of cognitive underlay two-way relay networks with interference and imperfect channel state information. J Wireless Com Network 2018, 53 (2018). https://doi.org/10.1186/s13638-018-1063-z

[13] W. Xu, J. Zhang, P. Zhang and C. Tellambura, "Outage Probability of Decode-and-Forward Cognitive Relay in Presence of Primary User's Interference," in IEEE Communications Letters, vol. 16, no. 8, pp. 1252-1255, August 2012, doi: 10.1109/LCOMM.2012.061912.120770.

[14] Sheng-nan YAN and Yan LI "Performance analysis of cognitive relay systems with spectrumsharing interference under a primary outage probability constraint", The Journal of China Universities of Posts and Telecommunications", vol. 21, number 1, pp. 16-21, 2014, https://doi.org/10.1016/S10058885(14)60263-6.

[15] S. S. Ikki and M. H. Ahmed, "Performance analysis of adaptive decode-and-forward cooperative diversity networks with best-relay selection," in IEEE Transactions on Communications, vol. 58, no. 1, pp. 68-72, January 2010, doi: 10.1109/TCOMM.2010.01.080080.

[16] J. Hu and N. C. Beaulieu, "Closed-Form Expressions for the Outage and Error Probabilities of Decode-and-Forward Relaying in Dissimilar Rayleigh Fading Channels," 2007 IEEE International Conference on Communications, Glasgow, 2007, pp. 5553-5557, doi: 10.1109/ICC.2007.920.

[17] Y. Han, S. H. Ting and A. Pandharipande, "Cooperative Spectrum Sharing Protocol with Selective Relaying System," in IEEE Transactions on Communications, vol. 60, no. 1, pp. 62-67, January 2012, doi: 10.1109/TCOMM.2011.100411.100469.

[18] I. Krikidis, H. A. Suraweera, P. J. Smith and C. Yuen, "Full-Duplex Relay Selection for Amplifyand-Forward Cooperative Networks," in IEEE Transactions on Wireless Communications, vol. 11, no. 12, pp. 4381-4393, December 2012, doi: 10.1109/TWC.2012.101912.111944.

[19] Blasco-Serrano, R., Lv, J., Thobaben, R. et al. Multi-antenna transmission for underlay and overlay cognitive radio with explicit message-learning phase. J Wireless Com Network 2013, 195 (2013). https://doi.org/10.1186/1687-1499-2013-195. 
[20] Zahedi, A. Performance analysis of multiple-relay cognitive communication system in Rayleigh fading channel. Telecommun Syst 72, 389-399 (2019). https://doi.org/10.1007/s11235-019-00574-8.

[21] A. Vashistha, S. Sharma and V. A. Bohara, "Outage Analysis of a Multiple-Antenna Cognitive Radio System With Cooperative Decode-and-Forward Relaying," in IEEE Wireless Communications Letters, vol. 4, no. 2, pp. 125-128, April 2015, doi: 10.1109/LWC.2014.2382587. 
Figures

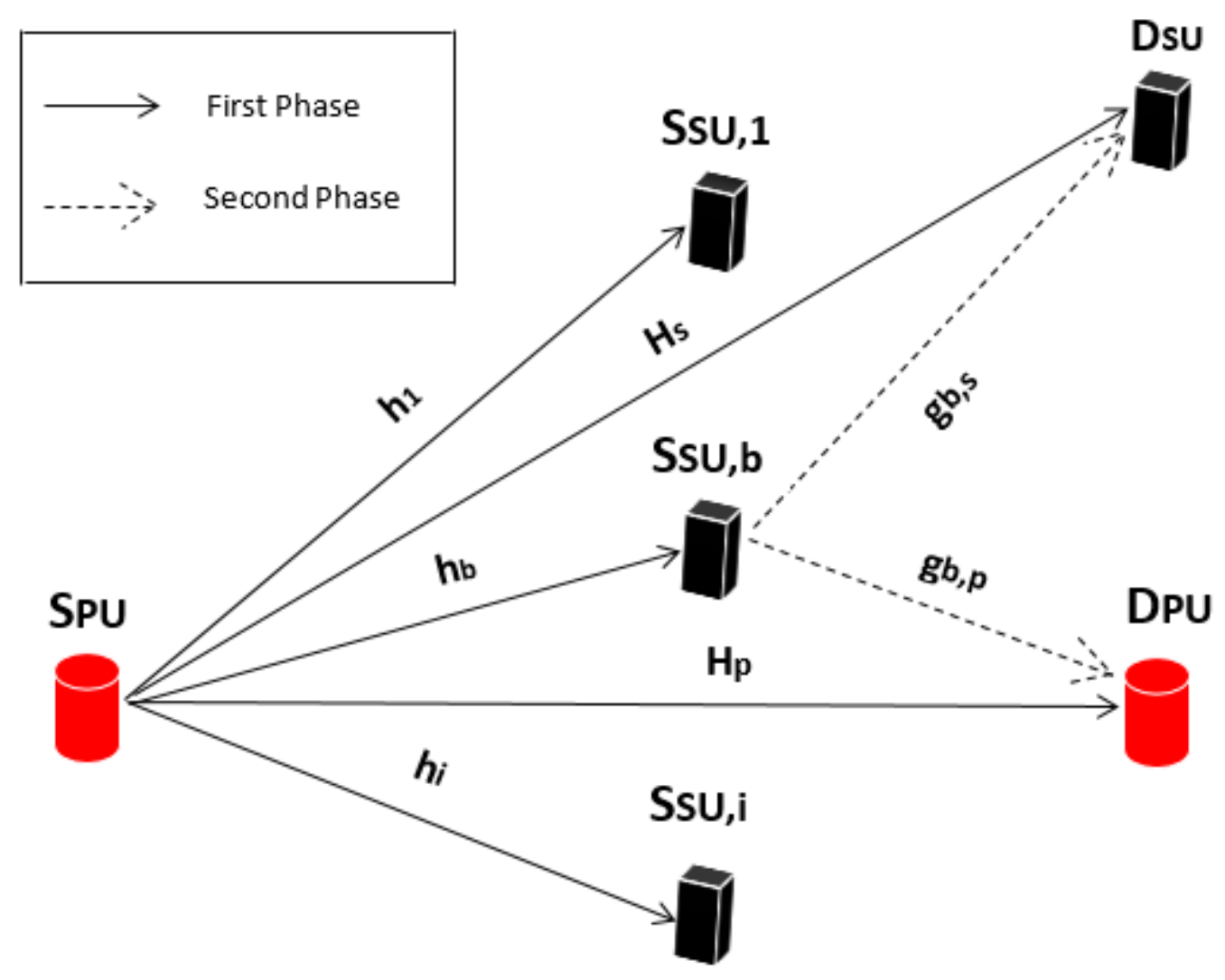

Figure 1

Proposed cognitive-based cooperative communication system 


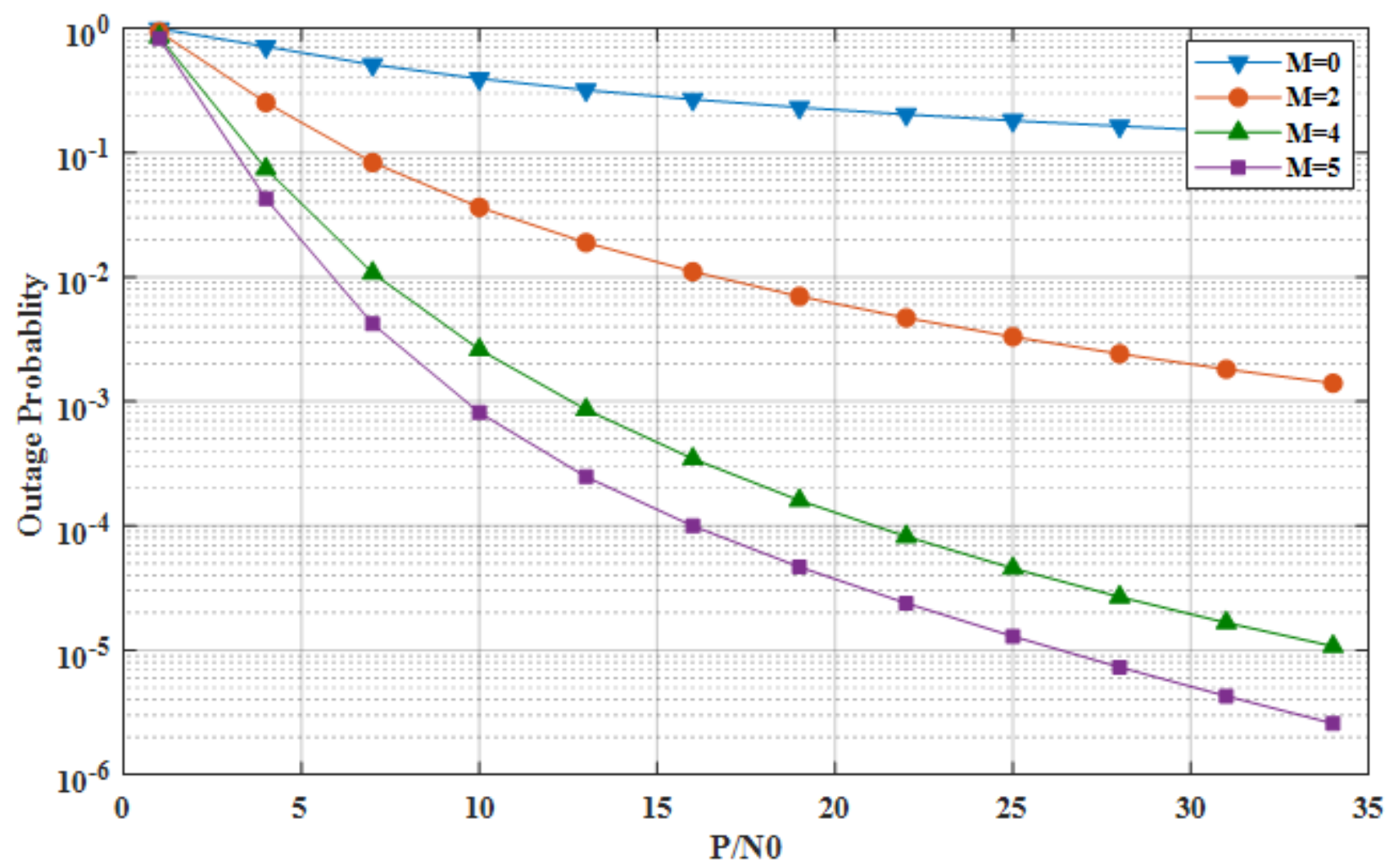

Figure 2

Outage probability of PU versus the SNR in different number of relays

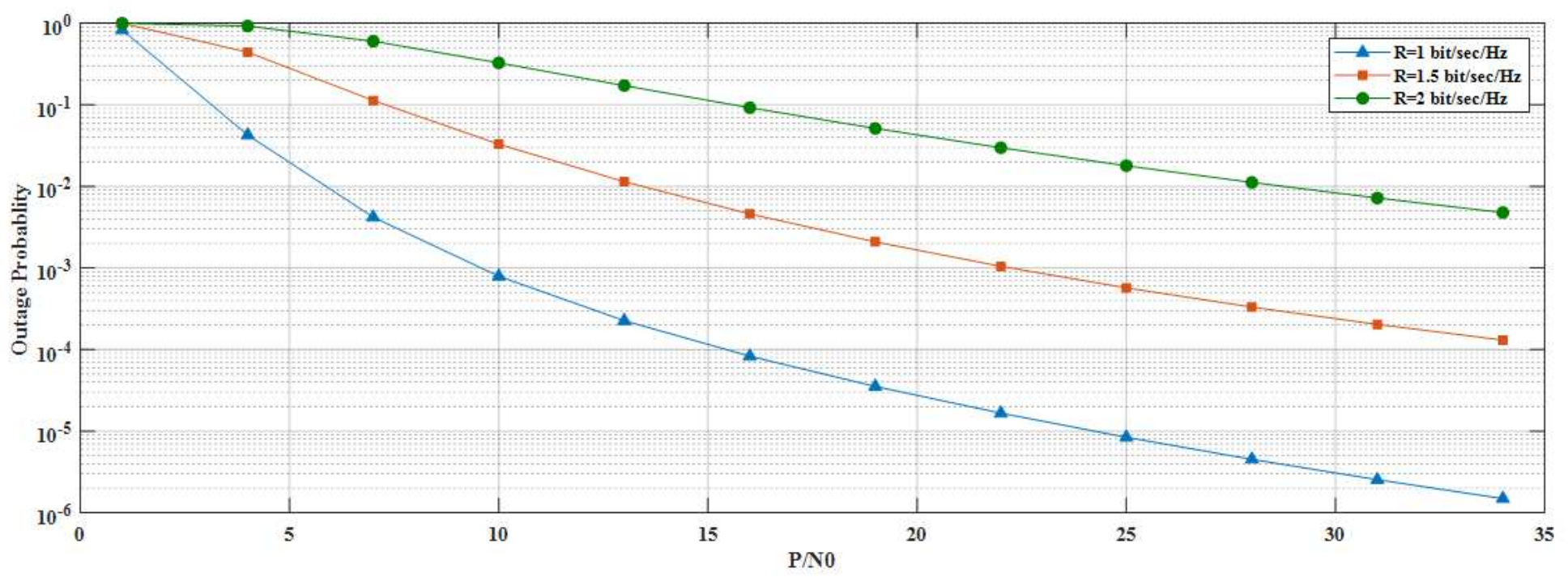

Figure 3

Outage probability of PU versus the SNR in different outage threshold 


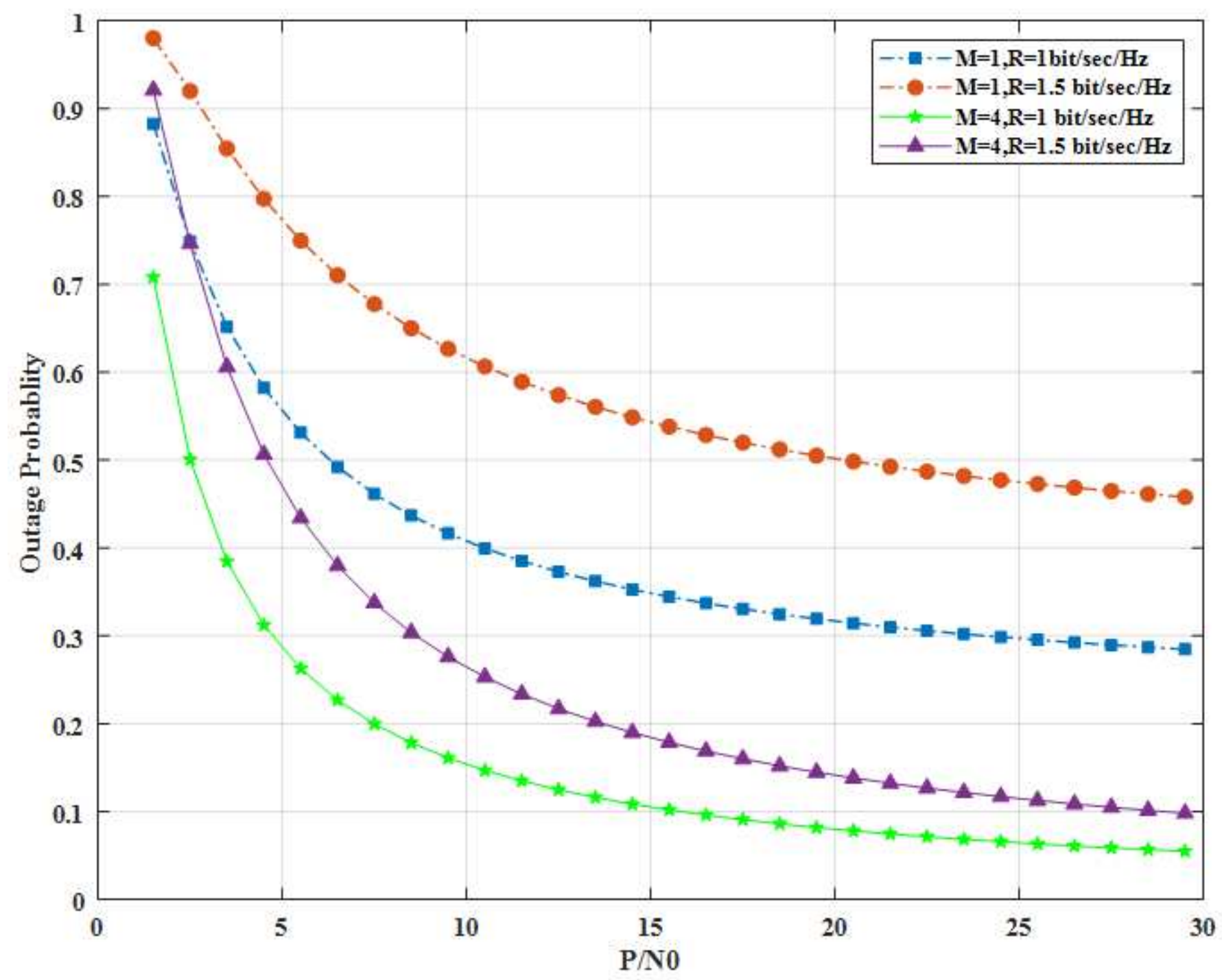

Figure 4

Outage probability of SU versus the SNR in different number of relays and outage thresholds 